\title{
Two Stage Fusion in Cognitive Radio Networks: A Clustering Approach
}

Rashid Mustafa*, Reena Rathee Jaglan, Sunil Agrawal

Department of ECE, U.I.E.T., Panjab University, Chandigarh 160014, India

Corresponding Author Email: mustafarashid80@gmail.com

https://doi.org/10.18280/mmep.070118

Received: 24 January 2019

Accepted: 12 January 2020

\section{Keywords:}

cognitive radio network, cooperative spectrum sensing, cluster head, neural network, probability of detection

\begin{abstract}
The cognitive radio (CR) has been recently proposed as an auspicious technology to effectively utilize the radio band to allow unlicensed users by allocating the spectrum dynamically on a non-interfering basis. The main challenge of the CR system is control channel overhead when the number of secondary users (SUs) becomes very large in cooperative spectrum sensing (CSS) scenario. To mitigate this problem, Cluster based CSS approach is useful to avoid the crowding on the control channel. Therefore, in this paper, neural network approach based on supervised learning algorithm is applied at the fusion center (FC) for proposed clustering technique. The simulation results prove that proposed clustering approach enhance the accuracy of the system. Also, it is more robust than other conventional schemes.
\end{abstract}

\section{INTRODUCTION}

In Cognitive Radio (CR) technology, the secondary users (SUs) utilized the primary user (PU) frequency band in an opportunistic manner. Performance of sensing will be degraded in the case of single user when the fading effect occurs. To overcome this issue cooperative spectrum sensing (CSS) has been proposed [1-3]. The current research has generally related on the fusion center (FC) based collaborative model in which the detecting hubs report their detecting results to the common base station through control channel employing soft or hard methodologies. In soft scheme, the entire test statistics of receive data is sent to the common base station. In hard scheme, the FC utilizes strategies such as ORrule [4], AND-rule [5, 6], K-out-of-N rules [7-9], or Machine

\section{Learning (ML) $[10,11]$.}

When the number of SUs becomes very large then the overloading problem occurs on the control channel, to mitigate this cluster based CSS is proposed [12]. In cluster-based CSS technique, cognitive radio users (CRs) are divided into two categories, as shown in Figure 1: Cluster head $(\mathrm{CH})$ and non$\mathrm{CH}$ member. In the clustering technique, all SUs individually detect the PU signal and send their results to $\mathrm{CH}$. The $\mathrm{CH}$ decides whether the PU signal is existing or not. The decision of the $\mathrm{CH}$ is the decision of the particular cluster in the SUs group, in the same way all CHs make the verdict about the PU presence and send their information to the FC. After receiving this information, FC uses a fusion rule and decides whether a $\mathrm{PU}$ is existing or not and sends its final decision to the SUs or non- $\mathrm{CH}$ members via $\mathrm{CH}$.

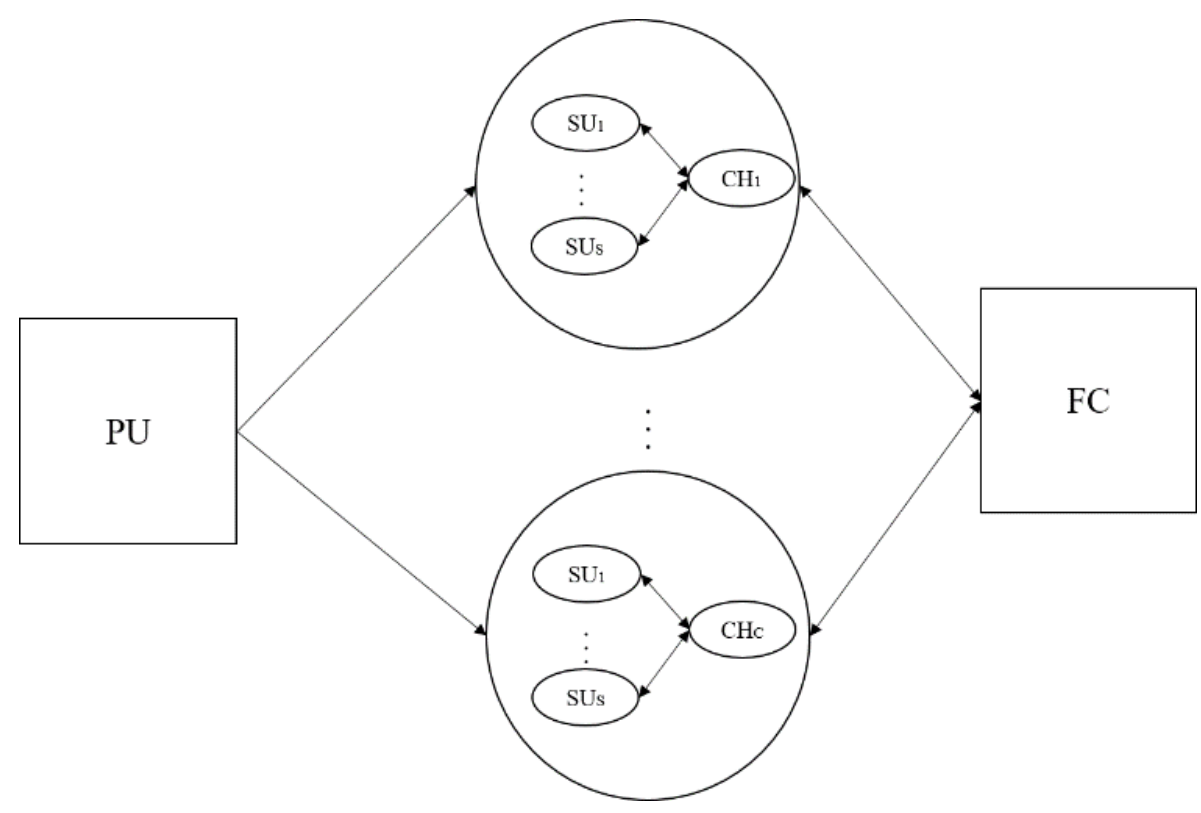

Figure 1. Block diagram of clustering scheme 


\section{RELATED WORK}

In case of the imperfect channel conditions, the cluster based CSS was proposed [13] using OR fusion rule. As compared to the conventional CSS, the detecting performance was enhanced to a great amount, but the spectrum efficiency was not taken into account. Guo et al. [14] used OR fusion rule to stabilize the cooperative overhead and sensing performance trade-off by optimizing the total number of SUs in a cluster. Also, the cluster architecture formation and $\mathrm{CH}$ selection was described by the authors. The overall impact of clustering technique is that it decreases the reporting errors and also the sensing performance was increased in comparison to the other sensing technique [15].

Reisi et al. [16] proposed a novel cluster-based algorithm to decrease the reporting channel bandwidth. In this method, CRs in each group sense the spectrum by the help of soft method and send the hard decisions to the FC Wang et al. [17] investigates cluster-based CSS issues in two-layer hierarchical CRNs with soft data fusion rule. Nguyen et al. [18], proposed a cluster-based optimal selective CSS scheme for reducing reporting time and bandwidth while preserving a certain level of sensing performance. At the FC, the Chair-Varshney rule has been used based on the clusters information to obtain a significant improvement in sensing performance [19].

In this paper, a neural network based clustering approach is applied at FC to make a reliable fusion decision.

The rest of the paper is organized as follows. Related work is discussed in section 2. A description of the system model is provided in section 3. Section 4 gives a description of the proposed clustering model. The results of the simulation are discussed in section 5. Finally, section 6 concludes the paper.

\section{SYSTEM MODEL}

\subsection{Cognitive radio network parameters}

Following parameters for CRN are used:

\section{- Cooperative Spectrum sensing scheme: Centralized}

- Spectrum sensing technique: Energy detection

- Number of SUs: 15 SUs

- Number of PU: 1 PU

- Decision making type: hard decision

\subsection{Mathematical model}

In the conventional CSS technique, $\mathrm{FC}$ received the energy information of all SUs, then makes a final decision about PU existence by comparing it with a pre-defined threshold [20]. The estimated energy metric is given as

$$
Y_{j} \approx\left\{\begin{array}{lll}
\chi_{2 u}^{2}\left(2 \gamma_{i, j}\right) & >\lambda & H_{1} \\
\chi_{2 u}^{2} & <\lambda & H_{0}
\end{array}\right.
$$

where, $Y_{j}$ is the energy statistics, $\lambda$ is detection threshold, $\chi_{2 u}^{2}$ is a chi-square distribution function and $\chi_{2 u}^{2}\left(2 \gamma_{i, j}\right)$ is a non-central chi-square distribution function with $2 u$ degrees of freedom (i.e., the time-bandwidth product $(u=W T)$ and $\left(2 \gamma_{i, j}\right)$ is the SNR of SU $j$ in cluster $i$ in $\left.\mathrm{dB}\right)$.
In the clustering scheme, the " $K$ " number of SUs divided into " $C$ " number of clusters. The number of SUs in the $i$-th cluster is denoted by " $S_{i}$ " and the $\mathrm{CH}$ signal to noise ratio (SNR) is represented by $\gamma_{i}$, which is highest signal strength received from the PU of the same cluster [13].

In clustering technique, each $\mathrm{SU}$ sends sensing information to $\mathrm{CH}$, then energy metric of $\mathrm{CH}$ can be given as

$$
G_{i} \approx\left\{\begin{array}{lll}
\chi_{2 S_{i} u}^{2}\left(2 \gamma_{i}\right) & >\lambda & H_{1} \\
\chi_{2 S_{i} u}^{2} & <\lambda & H_{0}
\end{array}\right.
$$

where, $\gamma_{i}=\sum_{j=1}^{S_{i}} \gamma_{i, j}$ is the instant SNR of the $\mathrm{CH}$ in the $i$-th cluster. The probability of detection $\left(Q_{d, i}\right)$ of cluster $i$ is represented by Digham et al. [21].

$$
Q_{d, i}=Q_{S_{i} u}\left(\sqrt{2 \gamma_{i}}, \sqrt{\lambda}\right)
$$

where, $Q_{s_{i} u}$ is the Marcum Q-function [22], then false alarm probability $\left(Q_{f a, i}\right)$ of cluster $i$ can be assumed as

$$
Q_{f a, i}=\frac{\Gamma\left(S_{i} u, \frac{\lambda}{2}\right)}{\Gamma\left(S_{i} u\right)}
$$

where, $\Gamma$ is gamma function [23].

\section{PROPOSED CLUSTERING SCHEME}

The proposed clustering schematic block diagram shown in Figure 1 comprises of a local decision at SUs stage and global decision at the FC stage. The energy detection process is done to make a local decision, i.e. sensing of the information regarding the channel availability. Also OR rule or NN are employed at the fusion stage to make a global decision. Moreover, a cluster based CSS approach is formulated in the global decision process, to prioritize spectrum utilization among all SUs in the current network based on their requirement. There are two approaches to cluster based CSS which are as follows:

\subsection{OR-OR fusion}

In this each SU individually senses the vacant band in a cluster by local sensing then sends this information to $\mathrm{CH}$. Then this information is sent to the FC. FC also gives its decision using OR rule.

Every $j \mathrm{SU}$ in cluster $i$ sends a local decision $G_{j}$ to the $\mathrm{CH}$ through energy detection technique

$$
G_{j}=\sum_{j=1}^{S_{i}} Y_{j} \begin{cases}\geq \lambda & 1 \\ <\lambda & 0\end{cases}
$$

where, $S_{i}$ is the number of SUs in the $i$-th cluster, which subject to $\sum_{i=1}^{C} S_{i}=K$ and $Y_{j}$ is energy decision statistics of SUs. 1 indicates PU existence and 0 indicates it's absent. 
The $\mathrm{CH}$ receives this local information in the same cluster and then make a cluster verdict $B_{i}$ through OR fusion rule

$$
B_{i}=\sum_{j=1}^{S_{i}} G_{j} \begin{cases}\geq 1 & 1 \\ <1 & 0\end{cases}
$$

Finally, the cluster verdicts for all the $\mathrm{CHs}$ are sent to the FC and then a concluding result is taken according to the following function

$$
H= \begin{cases}1 & \sum_{i=1}^{C} B_{i} \geq 1 \\ 0 & \sum_{i=1}^{C} B_{i}<1\end{cases}
$$

\subsection{NN-NN fusion}

NN approach is implementing through simulation framework dataset in which each SU decision used as input parameter and PU actual status is taken as target value to train the proposed fusion model, the Scaled Conjugate Gradient algorithm is used for training and the Sigmoid transfer function is used at output layer, after training generated model used as NN fusion model.

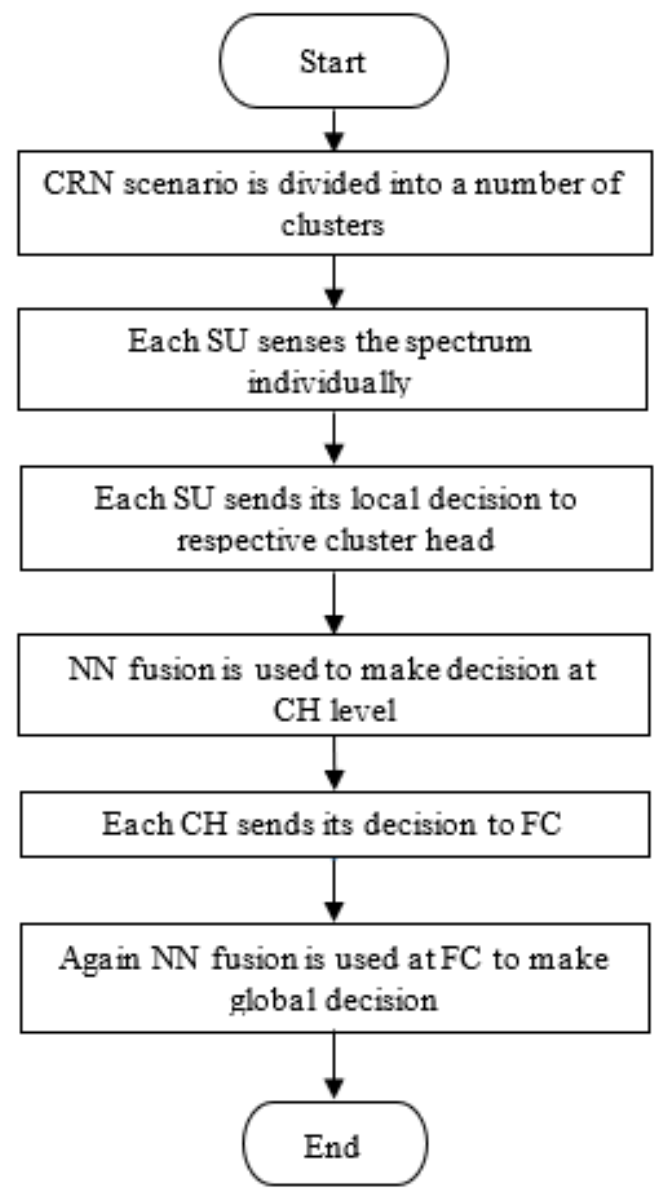

Figure 2. Flow diagram of proposed clustering scheme

In NN fusion, each SU individually senses the vacant band in a cluster by local sensing then sends this information to $\mathrm{CH}$. Then this information is sent to the FC. FC also gives their decision using NN fusion rule.

Every $j \mathrm{SU}$ in cluster $i$ sends a local decision $G_{j}$ to the
$\mathrm{CH}$ through energy detection technique, $G_{j}$ is given by (5).

The $\mathrm{CH}$ receives this local information in the same cluster and make a cluster and then make a cluster decision $B_{i}$ through NN fusion

$$
B_{i}= \begin{cases}1 & \sum_{j=1}^{S_{i}} w_{j} G_{j}>\lambda_{j} \\ 0 & \sum_{j=1}^{S_{i}} w_{j} G_{j}<\lambda_{j}\end{cases}
$$

where, $\sum_{j=1}^{S_{i}} w_{i} G_{j}, \lambda_{j}$ and $w_{j}$ are the $\mathrm{NN}$ output, threshold and weights respectively.

Finally, the cluster verdicts for all the $\mathrm{CHs}$ are sent to the FC and then a concluding result is taken according to the following function

$$
H= \begin{cases}1 & \sum_{i=1}^{C} B_{i} \geq \lambda_{i} \\ 0 & \sum_{i=1}^{C} B_{i}<\lambda_{i}\end{cases}
$$

where, $\lambda_{i}$ is the threshold.

The flow diagram in Figure 2 gives the implementation procedure of proposed clustering scheme with the use of NN fusion.

\section{RESULTS AND DISCUSSION}

A CR network scenario is considered, with one PU, one FC and number of SUs $=K$, having different SNR values from $20 \mathrm{~dB}$ to $15 \mathrm{~dB}$, and number of clusters $=C$

The simulation is done using MATLAB and results are evaluated and performance of the conventional OR scheme, OR based clustering scheme and proposed $\mathrm{NN}$ based clustering scheme are measured through receiver operating characteristics (ROC) curves and complementary ROC curves Table 1 shows the network parameters used for NN training.

Table 1. Essential network parameters used for training

\begin{tabular}{cc}
\hline Parameter & Value \\
\hline Network & Three Layer Back propagation \\
No. of inputs Layer & 1 \\
No. of hidden Layer & 1 \\
No. of output Layer & 1 \\
Hidden Layer Neurons & 10 \\
Training Algorithm & Scaled Conjugate Gradient \\
Training Function & Trainscg \\
\hline
\end{tabular}

\subsection{Performance analysis through ROC curves}

In Figure 3, the ROC curve shows the relationship between probability of detection $\left(Q_{d}\right)$ versus probability of false alarm ( $Q_{f a}$ ) of the conventional OR scheme, proposed OR-based and proposed NN-based clustering scheme. This plot shows that the proposed $\mathrm{NN}$ based clustering scheme performs better than the other schemes. In proposed NN based clustering approach accuracy is $98.5 \%$ while in the case of OR-based clustering scheme accuracy is $88.5 \%$ and in case of conventional OR scheme accuracy is $82.5 \%$. Also, false alarm probability of proposed scheme is less than the other schemes. 


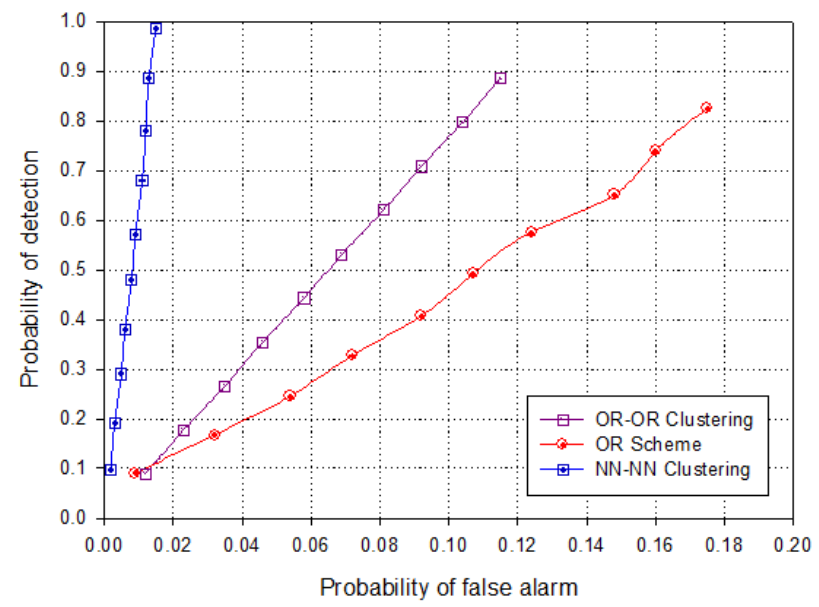

Figure 3. ROC performance

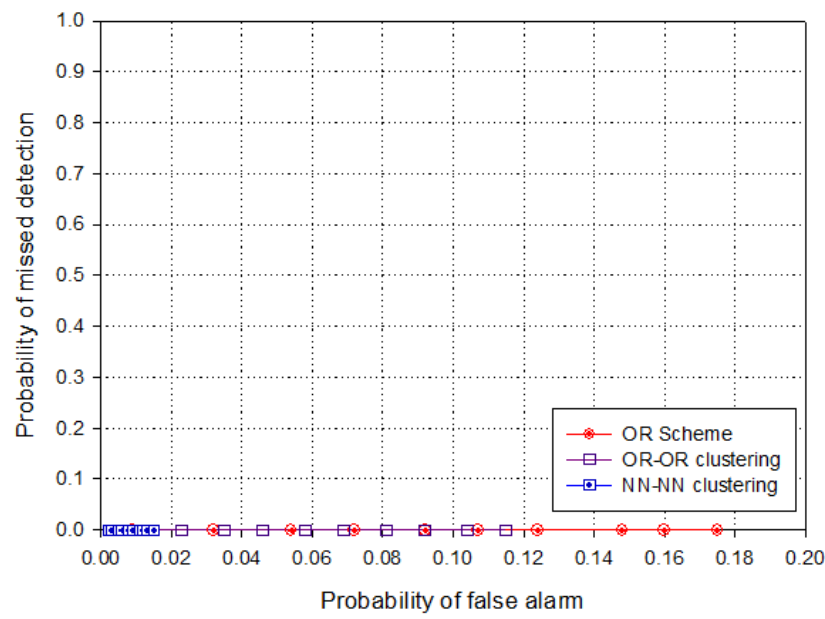

Figure 4. CROC performance

Figure 4 shows the complementary ROC performance curve, which shows the relation between the missed detection probability $\left(Q_{m d}\right)$ versus probability of false alarm $\left(Q_{f a}\right)$ of conventional OR scheme, OR-OR clustering scheme and proposed NN based clustering scheme. This plot shows that missed detection probability of the conventional OR scheme, cluster based OR-OR scheme and proposed NN based clustering scheme are almost negligible. In all schemes missed detection probability is zero.

In addition, it can be seen that the false alarm probability of the proposed $\mathrm{NN}$ clustering scheme is also the less than to the conventional OR scheme, cluster based OR-OR scheme at all values of miss-detection probability. This result shows that the proposed $\mathrm{NN}$ based clustering scheme performs better than the conventional CSS schemes.

Table 2. Performance metrics of different schemes

\begin{tabular}{ccc}
\hline Schemes & $Q_{d}$ & $Q_{f a}$ \\
\hline OR fusion & .825 & .175 \\
\hline OR-OR fusion & .885 & .115 \\
\hline NN-NN fusion & .985 & .015 \\
\hline
\end{tabular}

Table 2 shows the performance metrics of different fusion schemes and for better visualization its graphical representation is also given in Figure 5. From this analysis, it can be concluded that proposed clustering scheme gives best trade-off between different performance parameters.

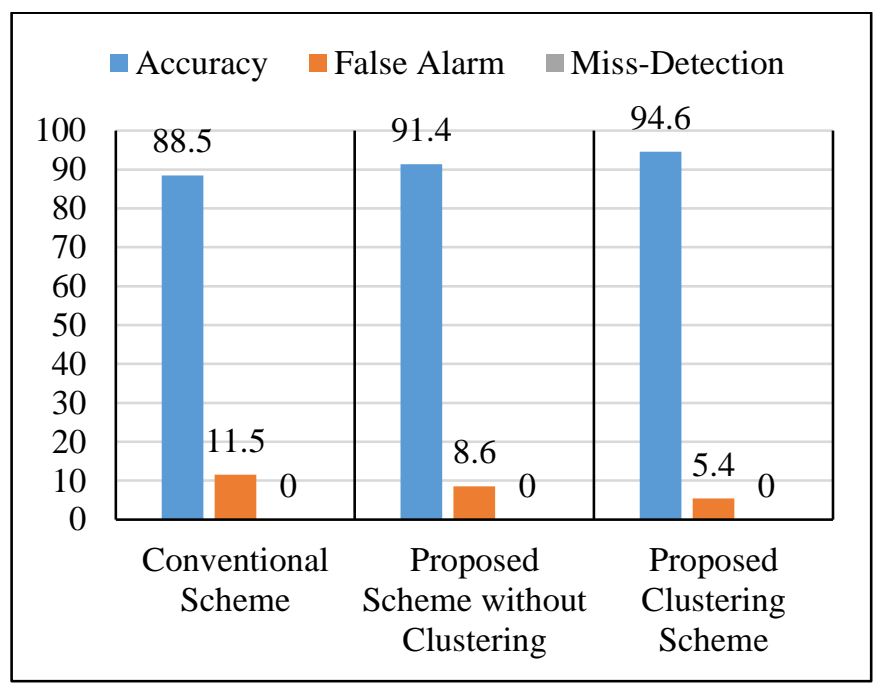

Figure 5. Graphical representation of different schemes

\section{CONCLUSIONS}

In this paper, the performance of $\mathrm{NN}$ based clustering scheme in cognitive radio network is investigated. The performance of different schemes are analysed through simulation. It has been evaluated from simulation results that performance of the proposed $\mathrm{NN}$ clustering scheme is much better as compared to conventional OR and OR-based clustering scheme. The results prove that proposed clustering approach enhance the accuracy and reduce the false alarm of the system by $10 \%$. Also, proposed approach shows that it is more robust than other conventional schemes.

\section{ACKNOWLEDGMENT}

We thank the Director of UIET (Panjab University) for providing the lab facility.

\section{REFERENCES}

[1] Akyildiz, I.F., Brandon, F.L., Balakrishnan, R. (2011). Cooperative spectrum sensing in cognitive radio networks: A survey. Physical Communications, 4(1): 4062. http://dx.doi.org/10.1016/j.phycom.2010.12.003

[2] Jaglan, R.R., Mustafa, R., Sarowa, S., Agrawal, S. (2016). Performance evaluation of energy detection based cooperative spectrum sensing in cognitive radio network. Proceeding of First International Conference on Information and Communication Technology for Information Systems, 2: 585-593. https://dx.doi.org/10.1007/978-3-319-30927-9_58

[3] Khan, S.M., Khan, W.M., Faraz, F.U., Khan, S.M. (2018). Incremental voting based spectrum sensing model for cognitive radio networks. Review of Computer

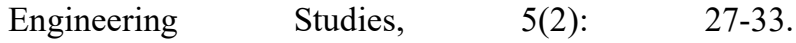
http://dx.doi.org/10.18280/rces.050201

[4] Umebayashi, K., Lehtomäki, J.J., Suzuki, Y. (2012). Study on efficient decision fusion in OR-rule based 
cooperative spectrum sensing. In Proceedings of the IEEE Wireless Communications and Networking Conference, Shanghai, China, pp. 714-718. https://doi.org/10.1109/WCNC.2012.6214464

[5] Visotsky, E., Kuffner, S., Peterson, R. (2005). On collaborative detection of TV transmissions in support of dynamic spectrum sharing. In Proceedings of the IEEE International Symposium on New Frontiers in Dynamic Spectrum Access Networks, Baltimore, MD, USA, pp. $338-345$ https://doi.org/10.1109/DYSPAN.2005.1542650

[6] Zheng, Y., Xie, X., Yang, L. (2009). Cooperative spectrum sensing based on snr comparison in fusion center for cognitive radio. In Proceedings of the International Conference on Advanced Computer Control, Singapore, $\quad$ pp. 212-216. https://doi.org/10.1109/ICACC.2009.52

[7] Chen, C., Cheng, H., Yao, Y.D. (2011). Cooperative spectrum sensing in cognitive radio networks in the presence of the primary user emulation attack. IEEE Transaction on Wireless Communications, 10(7): 21352141. https://doi.org/10.1109/TWC.2011.041311.100626

[8] Zhang, W., Mallik, R.K., Letaief, K.B. (2008). Cooperative spectrum sensing optimization in cognitive radio networks. In Proceedings of the IEEE International Conference on Communications, Beijing, China, pp. 3411-3415. https://doi.org/10.1109/ICC.2008.641

[9] Zhang, W., Mallik, R.K., Letaief, K.B. (2009). Optimization of cooperative spectrum sensing with energy detection in cognitive radio networks. IEEE Transaction on Wireless Communications, 8(12): 57615766. https://doi.org/10.1109/TWC.2009.12.081710

[10] Lee, W., Kim, M., Cho, D.H., Schober, R. (2019). Deep Sensing: Cooperative spectrum sensing based on convolutional neural networks. IEEE Transactions on Vehicular Technology, 68(3): 3005-3009. https://doi.org/10.1109/TVT.2019.2891291

[11] Ghasemi, A., Sousa, E.S. (2005). Collaborative spectrum sensing for opportunistic access in fading environments. In Proceedings of the IEEE DySPAN 2005, Baltimore, MD, USA, pp. 131-136 https://doi.org/10.1109/DYSPAN.2005.1542627

[12] Jiao, Y., Yin, P., Joe, I. (2016). Clustering scheme for cooperative spectrum sensing in cognitive radio networks. IET Communications, 10(13): 1590-1595. https://doi.org/10.1049/iet-com.2015.0865

[13] Sun, C., Zhang, W., Letaief, K.B. (2007). Cluster-based cooperative spectrum sensing in cognitive radio systems.
In 2007 IEEE International Conference on Communications, Glasgow, UK, pp: 2511-2515. https://doi.org/10.1109/ICC.2007.415

[14] Guo, C., Peng, T., Xu, S., Wang, H., Wang, W. (2009). Cooperative spectrum sensing with cluster-based architecture in cognitive radio networks. In IEEE 69th Conference on Vehicular Technology, VTC Spring 2009, Barcelona, Spain, pp: 1-5. https://doi.org/10.1109/VETECS.2009.5073471

[15] Wang, Y., Feng, C., Zeng, Z., Guo, C. (2009). A robust and energy efficient cooperative spectrum sensing scheme in cognitive radio networks. 2009 11th International Conference on Advanced Communication Technology, Phoenix Park, South Korea, pp. 640-645.

[16] Reisi, N., Jamali, V., Ahmadian, M., Salari, S. (2011). Cluster-based cooperative spectrum sensing in cognitive radio networks under log-normal shadow-fading. 2011 19th Iranian Conference on Electrical Engineering, Tehran, Iran, pp. 1-5.

[17] Wang, Y., Lin, W., Huang, Y., Ni, W. (2014). Optimization of cluster-based cooperative spectrum sensing scheme in cognitive radio networks with soft data fusion. Wireless personal communications, 77(4): 2871-2888. https://doi.org/10.1007/s11277-014-1673-7

[18] Nguyen, T.N., Koo, I. (2013). A cluster-based selective cooperative spectrum sensing scheme in cognitive radio. EURASIP Journal on Wireless Communications and Networking, 176(2013): 1-9. https://doi.org/10.1186/1687-1499-2013-176

[19] Hussain, M., Tripathi, P. (2017). A cluster based selective cooperative spectrum sensing technique for cognitive radio network. International Journal of Engineering and Management Research (IJEMR), 7(2): 8-12.

[20] Urkowitz, H. (1967). Energy detection of unknown deterministic signals. Proceeding of the IEEE, 55(4): 523-531. http://dx.doi.org/10.1109/PROC.1967.5573

[21] Digham, F.F., Alouini, M.S., Simon, M.K. (2007). On the energy detection of unknown signals over fading channels. IEEE Transaction on Communication, 55(1): 21-24. http://dx.doi.org/10.1109/TCOMM.2006.887483

[22] Nuttall, A. (1975). Some integrals involving the Q_M function (Corresp.). IEEE Transactions on Information Theory, 21(1): 95-96. http://dx.doi.org/10.1109/TIT.1975.1055327

[23] Gradshteyn, I.S., Ryzhik, I.M. (2007). Table of Integrals, Series, and Products. 7th edition, Academic Press, New York. p. 1200 